\title{
Mechanismen der Suchtentwicklung
}

Es gibt verschiedene Theorien, die eine Suchtentwicklung auf neuronaler und molekularer Ebene erklären. Es ist Konsens, dass Drogen zunächst über das Belohnungssystem ihre verstärkende Wirkung durch die Freisetzung von Dopamin im Nukleus accumbens (NAC) erzeugen. Wiederholter Drogenkonsum und die Darbietung drogenassoziierter Reize können sogar zu einer vermehrten Dopaminfreisetzung führen und einer damit verbunden psychomotorischen Stimulation. Dieser Sensibilisierungsprozess führt zu einer verstärkten Reiz-Attribution und Craving. Am Versuchstier wurden Sensibilisierungsprozesse überzeugend gezeigt, die entsprechenden Humanbefunde stehen jedoch noch größtenteils aus. Chronischer Drogenkonsum führt zu automatisierten Gewohnheiten; z. B. ist Rauchen ein hochautomatisiertes Verhalten - eine Person, die über 10 Jahre regelmäßig raucht, hat über eine Million Inhalationen durchgeführt. Diese automatisierten Gewohnheiten stehen mit einer Verschiebung vom ventralen zum dorsalen striatalen Prozessieren von drogenassoziierten Reize in Zusammenhang. Die Entwicklung von Gewohnheit wird durch eine Verringerung der frontostriatalen Kontrolle weiter befördert. Geminderte Top-down-Kontrolle ist ein Resultat neurotoxischer Drogeneffekte im präfrontalen Kortex und verwandten Hirnregionen nach chronischem Drogenkonsum. Verminderte kognitive Kontrolle kann ebenfalls durch neuroadaptive Ver-

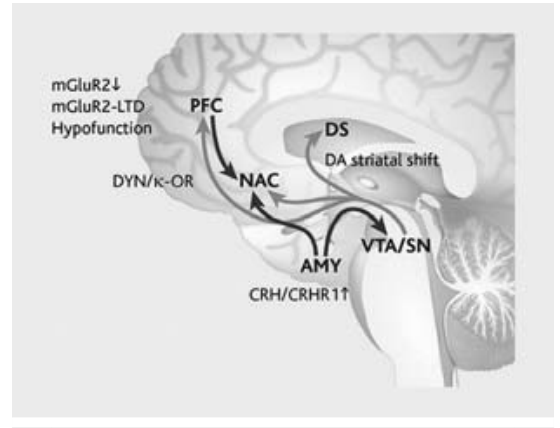

- Abb. 1 Ein phasisches Dopaminsignal im NAc führt zu Verstärkungsprozessen (Stimulus-Belohungslernen) und ,incentive salience" (Dopamin-Neurone sind schwarz gekennzeichnet). Im Übergang zu süchtigem Verhalten kommt es zu wiederholten Stimulus Reaktionsshemata die durch dorsostriatales Prozessieren vermittelt werden („striatal shift“. Die Top-down Kontrolle durch den PFC ist durch eine abgeschwächte Expression von mGluR2 verringert. Negativer Affekt bedingt sich durch eine Hochregulation des DYN/K-OR innerhalb des NAc und einem hyperaktiven $\mathrm{CRH} / \mathrm{CRHR} 1$ Systems in der Amygdala.

änderungen entstehen, die zu einer glutamaterg-GABAergen Dysbalance im präfrontalen Kortex (PFC) führen. Diese Dysbalance ist höchstwahrscheinlich das Ergebnis eines ausgeprägten Defizits im metabotropen Glutamatrezeptor-Subtyp 2 (mGluR2) - einem Hauptregulator des extrazellulären Glutamatspiegels - wie be- reits in Tiermodellen der Sucht und im menschlichen Gehirn von süchtigen Patienten gezeigt wurde. Darüber hinaus führt Neuroadaptation innerhalb des Belohnungs- und Stress-System zu einer reduzierten Wirkung von nicht-drogenassoziierten Reizen und alternativen Belohnungen sowie zu negativen Affekten und erhöhter Stress-Reaktivität. Diese Phänomene stehen mit einer verminderten tonischen striatalen Dopaminfreisetzung sowie einem hochregulierten DynorphinSystem (DYN) und extrahypotalamischen $\mathrm{CRH}$-System in Verbindung.

\section{Interessenkonflikte}

Finanzielle Kompensation für Vorträge und Beratung von Servier Deutschland und DA Pharma Paris.

Autor

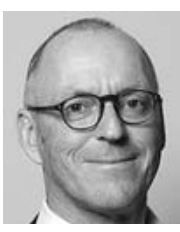

Rainer Spanagel Institut für Psychopharmakologie, Zentralinstitut für Seelische Gesundheit, Mannheim

Korrespondenzadresse

Prof. Dr. Rainer Spanagel

Zentralinstitut für Seelische Gesundheit Institut für Psychopharmakologie J 5

68159 Mannheim

rainer.spanagel@zi-mannheim.de 